FORMATION Formation emploi

Revue française de sciences sociales

98 | avril-juin 2007

Pour une approche par les capacités

\title{
Formation continue : quelles capacités d'action des salariés ? L'approche par les capacités comme grille de lecture
}

Continuing vocational training: what real opportunities do employees have? A study based on the capability approach

Weiterbildung: Welche Handlungsspielräume für Arbeitnehmer?A. Sens Konzept der Capabilities als Analyse-Raster

Marion Lambert et Josiane Vero

\section{(2) OpenEdition}

Journals

Édition électronique

URL : http://journals.openedition.org/formationemploi/1574

DOI : 10.4000/formationemploi. 1574

ISSN : $2107-0946$

Éditeur

La Documentation française

Édition imprimée

Date de publication : 1 avril 2007

Pagination : $55-75$

ISSN : 0759-6340

Référence électronique

Marion Lambert et Josiane Vero, « Formation continue : quelles capacités d'action des salariés ?

L'approche par les capacités comme grille de lecture », Formation emploi [En ligne], 98 | avril-juin 2007, mis en ligne le 30 juin 2009, consulté le 30 octobre 2020. URL : http://journals.openedition.org/ formationemploi/1574 ; DOI : https://doi.org/10.4000/formationemploi.1574 


\section{Numéro spécial}

\section{Formation continue: quelles capacités d'action des salariés? L'approche par les capacités comme grille de lecture}

Par Marion Lambert et Josiane Vero*

Les inégalités face à la formation continue sont importantes et déià bien connues. L'approche par les capacités permet d'éclairer les latitudes d'action des salariés et de dépasser l'opposition schématique entre formés et non-formés.

L'ambition de la loi de 1971 de faire de la formation un instrument de promotion sociale n'est que partiellement réalisée. On a certes assisté depuis cette période à un développement extensif de la formation. Cela étant, les objectifs de formation à vocation professionnelle visant l'adaptation des travailleurs aux changements des techniques et des conditions de travail l'ont emporté sur les enjeux civiques, sociaux et culturels (Berton et Podevin, 1991). Malgré la croissance des dépenses de formation, les travaux statistiques sur la formation continue ont largement commenté les inégalités de participation à la formation, en fonction du diplôme, de la catégorie socioprofessionnelle (Fournier et al., 2002; Fournier, 2004) du statut d'emploi (Goux et Maurin, 2000), de la trajectoire d'emploi (Perez et Thomas, 2004), de l'âge (Dupray et Hanchane, 2001; Lainé, 2003; Fournier, 2003), du sexe (Fournier, 2001). Au-delà des descripteurs individuels, les caractéristiques structurelles comme la taille et le secteur d'activité des entreprises permettent de dresser un panorama complet de ces inégalités (Géhin et Verdier, 1988; Gauthier et Marion, 2000 ; Lambert et Perez, 2004).

L'échec partiel des politiques de formation continue menées jusque-là a conduit les signataires de

* Marion Lambert est économiste, chargée d'études au Céreq dans le département « Production et Usages de la Formation Continue » (DFC). Ses travaux portent essentiellement sur la participation des salariés à la formation continue et la variété des modes de formation. Elle a notamment publié : Hanchane S. et Lambert M. (2003), " La variété des pratiques de formation : usages et enjeux ", Formation Emploi, $\mathrm{n}^{\circ} 81$, pp. 51-66 ou encore Lambert M. et Perez C. (2004), "Stratégies de recours et d'usage de la formation des "nouveaux recrutés": une comparaison publicprivé », in Les déterminants des entrées dans les fonctions publiques: parcours du combattant ou pis-aller? Paris, ministère de la Recherche, pp. 109-141. 
Josiane Vero est économiste, chargée d'études au Céreq dans le département "Production et Usages de la Formation Continue » (DFC). Ses travaux portent essentiellement sur l'application des théories de la justice à l'analyse de la pauvreté et à celle de la formation continue en entreprise. Elle a récemment publié : Vero J. (2006), « A comparison of poverty according to primary goods, capabilities and Outcomes. Evidence from French School Leavers' Surveys » in Betti et Lemmi (Éds), Fuzzy set approach to multidimensional poverty measurement, Springer, pp. 21 1-232 ou encore Perez C. et Vero J. (2006), "L'accès à la formation en entreprise au regard des modes de gestion de la main-d'œuvre », Travail et Emploi, juillet-septembre, $n^{\circ} 107$, pp. 59-71

Les auteurs sont toutes deux engagées dans le programme Européen de recherche Capright inscrit dans le 6e Programme-cadre pour la recherche et le développement (PCRD). Ce programme mobilise notamment l'approche par les capacités pour analyser la dynamique des relations entre la formation initiale et continue, l'emploi et la protection sociale.

1'Accord national interprofessionnel, du 20 septembre 2003, relatif à l'accès des salariés à la formation tout au long de la vie professionnelle - et repris dans la loi du 4 mai 2004 - à refonder la participation des salariés à la formation en entreprise. En effet, la récente loi entend organiser un droit négocié dans l'entreprise, encadré ou non par des accords collectifs, afin de "permettre à chaque salarié d'être acteur de sa formation $»^{1}$. Dès lors, s'interroger sur les capacités d'action des salariés vis-à-vis de la formation en entreprise devient central.

Mais peu d'études portent sur cette thématique. La formation continue est souvent étudiée au regard de l'accès des salariés, mais elle est plus rarement envisagée en amont de cet accès (Charlon-Dubar et al., 1990 ; Fournier, 2004 ; Perez et Vero, 2006) 2.

\footnotetext{
${ }^{1}$ Pour les actions de formation visant le « développement des compétences des salariés ", l'employeur a ainsi la possibilité d'inciter le salarié à partir en dehors du temps de travail (en lui versant une allocation correspondant à $50 \%$ de la rémunération nette de référence) ; le salarié, quant à lui, a le droit d'exiger un engagement écrit de l'employeur sur l'impact de la formation (accès prioritaire à certaines fonctions, attribution d'une classification).

${ }^{2}$ L'étude «Regards croisés sur la formation continue», initiée en 2003 par le Céreq et la Dares et menée en collaboration avec le laboratoire Travail et Mobilités (Paris X), fait figure d'exception (Gélot 2006, Personnaz et Vernoux-Marion, 2006, Perez et Vero, 2006).
}

Or, une analyse fondée sur les taux d'accès à la formation comporte en germes de nombreux conflits d'interprétation sur les marges de manœuvre des salariés et le partage des responsabilités dans le résultat de l'accès ou du non-accès à la formation. Faute d'une clarification des responsabilités dans ce champ, les malentendus demeurent. Ainsi, des interprétations contradictoires s'opposent entre, d'une part, ceux qui invoquent l'absence «d'appétence » de certaines catégories de salariés pour la formation, et d'autre part, ceux qui, sans attendre nécessairement que les entreprises se comportent comme un service public de formation continue, invoquent la responsabilité des entreprises qui ne configureraient pas un même champ des possibles pour toutes les catégories de salariés. À partir de l'enquête FC2006 sur la formation continue, complémentaire à l'enquête Emploi (encadré 1), nous avons souhaité remonter aux capacités d'action des salariés afin d'analyser leur latitude d'action face aux divers modes de formation et de reconsidérer la question de leur « appétence ». Ainsi, à la veille de la mise en application des dispositions de la nouvelle loi sur la formation, quel est le nombre et la nature des opportunités de formation offertes aux salariés? Quelles sont les contraintes auxquelles elles sont associées ? Comment les salariés s'emparent-ils du champ des possibles, caractérisé par une articulation de contraintes et d'opportunités ?

Par sa focalisation sur les latitudes d'action offertes aux salariés, ce travail mobilise l'approche par les capacités (Sen, $1985 ; 1992 ; 1999$ ), afin de porter un regard différent sur la formation continue. Il conviendra d'abord de préciser ses intentions et de les développer. Puis dans un second temps, nous présenterons les résultats d'une classification fondée sur l'ensemble des possibles offerts aux salariés. Cela nous permettra, ensuite, d'analyser la correspondance que l'on peut établir entre capacité d'action et qualification des emplois, diversité des modes de formation et contexte d'entreprise, avant d'ouvrir le débat sur la question de l'appétence. Enfin, pour conclure, nous nous interrogerons sur les enjeux d'une approche par les capacités au regard des nouvelles dispositions introduites par la loi du 4 mai 2004. 


\section{Encadré 1 \\ L'enquête Formation Continue 2000 (FC2000)}

L'enquête Formation Continue est une enquête complémentaire à l'enquête Emploi de l'Institut national de la statistique et des études économiques (INSEE), de mars 2000, coréalisée par le Céreq et I'INSEE (*). L'enquête FC2000 a été réalisée, en mars 2000, auprès d'un échantillon de 28700 personnes, âgées de moins de 65 ans, qui avaient achevé leur formation initiale et n'effectuaient pas leur service national au moment de l'enquête. Chacune d'elle a décrit l'ensemble des formations suivies entre janvier 1999 et février 2000, après la sortie du système scolaire, quelle qu'ait été la finalité de ces formations (professionnelle, sportive, sociale ou culturelle). L'enquête a été menée sous la forme d'entretiens en face-à-face portant sur les formations suivies. Des informations relatives aux formations suivies entre mars et décembre 1998 ont également été recueillies. L'enquête offre donc un recul important permettant d'apprécier les retombées des formations suivies au cours d'une période de deux ans.

Cette enquête complète les informations existant par ailleurs : enquêtes décennales sur la formation et la qualification professionnelle (FQP), enquêtes permanentes sur les conditions de vie des ménages, déclarations annuelles administratives obligatoires des entreprises (formulaires 24-83).

Le champ retenu dans cet article est celui des salariés du public et du privé (17 104 observations représentant environ 19502000 salariés). Plus d'un tiers des salariés ont suivi au moins une formation entre janvier 1999 et février 2000. Parmi l'ensemble des actions de formation suivies, 80 \% visaient d'abord une adaptation à l'emploi et $78 \%$ étaient principalement financées par l'employeur.

(*) Elle a été conçue dans le cadre d'un partenariat entre l'INSEE, la Direction de l'Animation de la recherche des Études et des Statistiques (DARES), la Direction générale de l'Emploi et de la Formation professionnelle (DGEFP) et le Service des Droits des femmes et de l'Égalité (SDFE) du ministère de l'Emploi, la Direction de la Programmation et du Développement du ministère de l'Éducation nationale (DPD), le Commissariat général du Plan (CGP) et le Céreq.

\section{L'APPROCHE PAR LES CAPACITÉS}

L'origine de l'approche par les capacités remonte aux discussions en philosophie politique qui ont connu une embellie après la parution, en 1971, de l'ouvrage de Rawls, "Théorie de la Justice». Ce débat a suscité des réponses variées à la question centrale «égalité de quoi?». Dans sa Tanner Lecture on Human Values, en 1979, intitulée «Equality of what? », Sen argumente pour sa part en faveur de la capabilité ${ }^{3}$ à fonctionner, c'est-à-dire l'éventail de ce qu'une personne peut faire et être ${ }^{4}$. Depuis, l'approche par les capacités a été largement développée à la fois par Sen et d'autres chercheurs, tant dans ses fondements théoriques que sur la question de son opérationnalité, pour devenir aujourd'hui un

\footnotetext{
${ }^{3}$ Le concept de « capabilities » de Sen est traduit en français indistinctement par « capacités » ou par le néologisme « capabilités ». ${ }^{4}$ Ce texte a été publié en 1980. $C f$. la bibliographie.
}

cadre normatif mobilisé à la fois pour l'évaluation des situations individuelles ou des arrangements sociaux, la mesure des inégalités ou la réflexion sur les politiques publiques.

\section{Des concepts théoriques...}

Ainsi, l'évaluation des situations des personnes et des politiques publiques doit se réaliser sur la base d'information des «capabilités » ${ }^{5}$, c'est-à-dire des libertés réelles des personnes de mener à bien des projets auxquels elles accordent de la valeur. L'approche par les capacités, se référant explicitement à Marx, vise à donner un contenu réel, au-delà

\footnotetext{
${ }^{5}$ «La base d'information sur laquelle se fonde un jugement identifie les informations dont dépend directement le jugement et, de façon non moins importante, affirme que le caractère vrai ou faux de tout autre type d'informations ne peut pas directement influer sur la justesse du jugement. La base d'information requise pour juger la justice délimite ainsi l'ensemble des faits auxquels s'appliqueraient directement les considérations en matière de justice » (Sen, 1999, pp. 215).
} 
des aspects formels, à l'idée de liberté. Il s'agit d'offrir à chacun les moyens d'orienter sa vie et son travail comme bon lui semble, dans la limite des ressources que la société peut mobiliser à cet effet. Trois concepts centraux sont à la base de l'approche par les capacités : les ressources, les capabilités et les fonctionnements.

Les «ressources» représentent les biens et services, produits ou non sur le marché, dont dispose une personne ( $C f$. Schéma 1 de Bonvin et Farvaque dans ce numéro, page 12). Par extension, sont inclus aussi les droits formels à la formation. Mais pour Sen, ces ressources ne sont pas considérées pour elles-mêmes mais par rapport aux fonctions qu'elles sont a priori appelées à remplir6. Bonvin (2005) résume cette idée en parlant de «ressources pour l'action ». En matière de formation continue, le passage d'un cadre régi par la loi de 1971 à celui de 2004 a profondément modifié les ressources pour l'action dont disposent les salariés. Jusqu'à l'adoption de la loi du 4 mai 2004, à côté du Congé individuel de formation (CIF), à l'initiative du salarié, n'existait qu'une obligation de dépenses pour les entreprises ou à défaut un versement au trésor public et en aucun cas un droit, même formel, à la formation. Par ailleurs, la jurisprudence et la loi Aubry II avaient créé une obligation d'adaptation des salariés à l'évolution de leur emploi. Mais le droit des salariés ne résultait que des obligations faites aux entreprises. Le basculement introduit par la loi de 2004, avec le DIF (droit individuel à la formation), nouveau droit formel dont disposent les salariés, constitue un élargissement des ressources.

L'ensemble de ce qu'un individu peut faire et peut être réellement, compte tenu des ressources dont il

\footnotetext{
${ }^{6}$ La présentation faite par Sen (1985) repose à la base sur la théorie du consommateur élaborée par Gorman (1956) et Lancaster (1966), laquelle envisage les biens en fonction de leurs caractéristiques, c'est-à-dire par rapport aux diverses utilisations qui peuvent être dérivées de la consommation d'un bien. Ainsi, Sen emprunte à Gorman et Lancaster la distinction entre d'une part, les biens et, d'autre part, les caractéristiques des biens. Pour illustrer cette différence, Sen $(1985$, p. 6) fournit l'exemple d'un bien, la nourriture, et de ses diverses caractéristiques : «La possession de nourriture procure à son détenteur l'accès aux propriétés de la nourriture, comme assouvir la faim, assurer la nutrition, offrir un plaisir gustatif, ou apporter un support aux relations sociales. »
}

dispose, est appelé ses «capabilités» par Sen et représente sa liberté réelle. Ce que traduit avant tout la capabilité, c'est la liberté de choix dont jouit une personne dans sa vie et son travail, que l'on appellera aussi son champ des possibles. Ici, la liberté réelle s'articule autour de deux dimensions : l'une est liée aux «opportunités » et renvoie à la nature et au volume des options réellement disponibles, et l'autre a trait au «processus» par lequel les décisions personnelles sont mises en œuvre et sont en mesure de jouer sur les choix. L'intérêt d'articuler ces deux dimensions est fondé sur l'hypothèse d'une dépendance forte entre le principe de participation actif et informé aux processus délibératifs et le développement des opportunités. Rapportée à l'analyse de la formation en entreprise, l'approche par les capacités amène à s'interroger sur les opportunités réelles de choix de formation dont dispose un salarié. Elle place aussi au cœur du débat la possibilité effective pour les salariés d'exprimer leur point de vue et de le faire entendre. Sont donc en jeu les procédures concrètes garantissant l'accès à la délibération, l'accès réel à l'information et la possibilité effective de peser sur la décision de formation (Perez et Vero, 2006).

La personne qui dispose ainsi d'un certain champ des possibles va être amenée à choisir certains états et certaines actions. Dans le vocabulaire de Sen, elle choisit d'accomplir certains «fonctionnements». Ainsi, la capabilité est définie comme «un ensemble de vecteurs de fonctionnements, qui indique qu'un individu est libre de mener tel ou tel type de vie » (Sen 1992, p. 66). Autrement dit, les capabilités incluent les fonctionnements, c'est-à-dire les comportements effectifs, mais elles ne s'y réduisent pas. Ainsi, "l'ensemble capabilité contient, entres autres, l'information sur la combinaison de fonctionnement réelle, celle qui a été choisie, puisqu'elle aussi, bien évidemment fait partie des combinaisons possibles » (Sen, 1992, p. 80). Si nous nous intéressons à la formation continue d'un salarié qui suit un stage de bureautique, l'accès à ce stage est en soi un élément de la capabilité de formation, puisqu'il fait partie de la combinaison réelle de fonctionnements. Mais l'ensemble «capabilité » nous fournit plus d'informations. Il contient aussi l'information complémentaire sur l'ensemble des autres choix possibles. 
Si les concepts de ressources et de fonctionnements sont reliés à la capabilité, il est important d'envisager leurs différences sous deux angles distincts.

Premièrement, les ressources n'indiquent pas ce que la personne est en capacité de faire quand elle en dispose. Il faut aussi qu'elle puisse les mobiliser et que le contexte social et environnemental permette cette mobilisation. Ainsi l'ouverture du DIF n'augmente en rien la capabilité de se former si le salarié n'est pas informé de son existence ou si les rapports de négociation avec son employeur ne sont pas équilibrés, même si l'instauration du DIF crée de nouvelles conditions de négociation. La capabilité à se former des personnes dépend donc autant de la possession de ressources, et donc en particulier des droits formels, que de la présence de facteurs de conversion favorables, qu'ils soient personnels (tels que le niveau de formation initiale ou de qualification, par exemple), sociaux (comme par exemple les garanties collectives ${ }^{7}$ ) ou environnementaux (par exemple les objectifs de l'entreprise, son organisation du travail, sa stratégie de gestion de la main-d'œuvre ou sa politique de formation).

S'agissant des fonctionnements, ils ne renseignent que partiellement sur les capabilités des personnes. Un même fonctionnement peut résulter de la présence ou de l'absence de capabilités. Par exemple, quelqu'un qui choisit de ne pas se former et qui refuse toutes les opportunités de formation proposées par son employeur, sans aucune contrainte de la part de celui-ci, jouit de la capacité réelle de se former dans la mesure où il dispose d'opportunités de formation en quantité suffisante et qu'il ne subit aucune contrainte à cet égard. Dans ce cas précis, on pourrait invoquer un manque d'appétence pour la formation. Dans le cas contraire, si le fait de ne pas se former est contraint par un contexte de pénurie d'opportunités de formation proposées par son employeur, la personne n'a pas de capabilité pour la formation: le salarié ne peut apporter sa contribution active et informée à la décision de formation.

${ }^{7}$ Pour une analyse de l'influence des garanties collectives, voir notamment Perez et Vero (2006) et Zimmermann (2006).
Ainsi, l'objectif de l'analyse de la formation continue en termes de capabilités est de rendre compte du champ des possibles du salarié en matière de formation. Par définition, les capabilités d'une personne en matière de formation continue, sur une année donnée, incluent les formations auxquelles elle a eu accès au cours de l'année, mais elles ne s'y réduisent pas. Les capabilités sont supposées rendre compte aussi de toutes les formations que l'entreprise a proposées au salarié, y compris celles qui ont été refusées par ce dernier, de tous les besoins non satisfaits, dont des demandes de formations refusées par l'employeur, des formations qui ont été imposées. Ainsi, l'analyse empirique des capabilités suppose de reconstituer, pour chaque personne, l'ensemble des alternatives concrètes qui s'offrent à elle, c'est-à-dire l'étendue des possibilités réelles - et non formelles qui lui sont offertes.

Le concept de capabilités est ici essentiel. On parle aussi indistinctement de liberté réelle, de latitude d'action, de capacité d'action, d'opportunité réelle de choix, voire de champ des possibles. Étant donné la polysémie de ce concept, le risque est grand de voir, dans l'approche par les capacités de Sen, un «réhabillage » d'approches existantes. En particulier, on pourrait y trouver une proximité avec "l'univers des formations possibles » évoqué par Charlon-Dubar et al. (1990), "l'univers des croyances", "le monde des possibles » analysés par Demazière et Dubar (1997). Au-delà de la proximité dans le vocabulaire utilisé, les deux positions se distinguent par au moins une importante différence. Contrairement à l'approche des sociologues que nous venons d'évoquer, pour Sen, il s'agit d'éviter une base d'information purement subjective fondée sur l'appréciation des personnes quand à leurs marges de manœuvre. En effet, fonder l'analyse sur l'appréciation des personnes pour évaluer leur propre situation soulève la question des «préférences adaptatives », selon laquelle la personne va adapter ses préférences aux contraintes qu'elle perçoit. Aussi, pour Sen, les indicateurs objectifs tiennent une place centrale. Ce point est essentiel chez Sen. Au contraire, dans les autres approches citées, la définition du champ des possibles n'est réservée qu'à la personne ellemême et de ce fait apporte une contribution intéressante, notamment pour la recomposition des logiques identitaires. 


\section{... à la mesure des capabilités}

Quelles sont les implications méthodologiques du passage d'une logique d'accès à une logique de capabilités? Par rapport à la première, l'approche par les capacités implique un basculement de l'objet sur lequel évaluer la situation des personnes. Existet-il, dès lors, un moyen d'élargir, de manière cohérente, l'information?

La multiplicité des informations sur des situations hypothétiques que suppose le cadre des capacités puisqu'il attache autant de valeur au comportement effectif qu'aux options que la personne a la capacité de choisir - est évidemment embarrassante pour une enquête empirique. Ces difficultés sont d'ailleurs reconnues par Sen. Comme le souligne Le Clainche (1994), alors que Sen s'évertue à développer une formalisation mathématique, il ne fournit pas une manière précise d'appliquer le concept ${ }^{8}$.

En substance, le premier problème dans l'évaluation des capabilités consiste à établir une sorte de liste de possibilités alternatives au comportement effectif (accès à tel type de formation, ou non-accès). Est donc en jeu le niveau de précision des données disponibles. Mais pour Sen (1992), l'approche par les capacités peut être utilisée à divers niveaux de précision. Idéalement, l'approche par les capacités supposerait d'examiner toute l'étendue de la liberté de choisir. Mais à défaut, il est parfois possible de creuser la notion de choix à partir des fonctionnements observés afin de parvenir à «une vision partielle mais significative des libertés dont jouissent des individus différents. » (Sen, 1992 p. 79). Il s'agit alors de représenter le fonctionnement observé sous un angle tel qu'il renseigne déjà sur certaines alternatives. C'est ce que Sen appelle un fonctionnement affiné : "Il est possible, de fait, de représenter les fonctionnements sous un angle tel qu'ils reflètent déjà les possibilités offertes, et donc les choix dont on bénéficie. Par exemple "jeuner" en tant que fonctionnement n'est pas purement et simplement être affamé. C'est choisir d'être affamé quand on a d'autres options. » (Sen, 1992, p. 82) Il est donc

${ }^{8}$ Sen fournit toutefois des moyens d'évaluer les capabilités dont jouissent les individus, mais ceux-ci restent globaux, mesurant à l'échelle d'une nation l'espérance de vie selon le sexe, la mortalité infantile, le taux d'analphabétisme, le niveau d'éducation, etc. (PNUD, 1998). possible, en pratique, de prendre en compte des choix en se contentant de relier les fonctionnements observés au réseau d'opportunités et de contraintes auquel il est associé, au lieu d'essayer d'introduire toute l'étendue de la liberté de choisir entre différents agencements de fonctionnements.

En outre, qui peut rendre compte des capabilités d'une personne? Pour Sen, l'appréciation des personnes sur leur situation compte, mais l'évaluation des capacités ne saurait s'y réduire. À côté de l'information subjective, des événements factuels ou «objectifs » nécessitent d'être considérés, notamment par l'observation d'un tiers extérieur. Ainsi, incorporer le point de vue des employeurs, salariés et représentants du personnel dans l'évaluation du champ des possibles des salariés face à la formation, apparaît alors comme une méthode $a d h{ }^{9}{ }^{9}$. Disposer d'enquêtes couplées « employeur - salariés » croisant les différents points de vue constituerait un net progrès. Dans l'attente d'une telle source statistique française ${ }^{10}$, l'exploitation de l'échantillon des salariés de l'enquête FC2000 nous a paru susceptible de fournir d'appréciables informations.

\section{QUELLES CAPACITÉS D'ACTION POUR QUELS MODES DE FORMATION ?}

La méthode que nous avons retenue ici se base sur les fonctionnements affinés. Ce choix nous a conduit à prendre appui sur certains fonctionnements et à inclure, dans leur description, une information sur les possibilités de choix et l'espace de liberté des personnes.

\footnotetext{
${ }^{9}$ L'étude « Regards croisés sur la formation continue », initiée en 2003 par le Céreq et la Dares, et menée en collaboration avec le laboratoire Travail et Mobilités (Paris X), nous fournit un bon exemple de l'adéquation de ce matériau à l'analyse des capacités d'action des salariés dans la discussion autour du départ en formation (Perez et Vero, 2006).

$\mathrm{Si}$ ces travaux permettent d'analyser finement les capacités d'action des salariés au regard des objectifs annuels de l'entreprise, de l'organisation du travail, des modes de gestion de la main-d'œuvre ou de la politique de formation, encore faut-il pouvoir quantifier à une échelle plus large les phénomènes étudiés.

${ }^{10}$ Le Céreq, en collaboration avec l'Insee et la Dares, prépare le croisement entre deux enquêtes sur la formation, l'une auprès des salariés (Formation continue 2006) et l'autre auprès des entreprises qui les emploient (Continuing Vocational Training Survey 3 ).
} 
En pratique, les capacités d'action du salarié face à la formation sont ici étudiées au travers de deux dimensions, les opportunités offertes par l'employeur d'une part, et les contraintes qui s'imposent à lui, d'autre part. Par opportunités, on entend les offres de formation proposées au salarié ; elles incluent les formations réalisées et celles que l'entreprise a proposé au salarié et qu'il a refusées. Par contraintes, on entend les offres de formations imposées au salarié. La présence de besoins de formation non satisfaits est également un marqueur des latitudes d'action. Nous avons donc aussi mobilisé cette information, malgré l'ambiguïté que soulève son interprétation ${ }^{11}$.

\footnotetext{
${ }^{11}$ Si l'absence de besoins de formation non satisfaits semble $a$ priori dénoter de fortes opportunités, les travaux de Fournier (2004) ont largement commenté le fait qu'elle allait de pair avec un taux d'accès à la formation faible. On repère peut-être ici un phénomène de "préférences adaptatives », où la résignation peut en effet pousser l'individu à adapter ses aspirations en matière de formation aux contraintes qu'il perçoit et donc à renoncer de manière inconsciente à émettre des besoins de formation pour se mettre en conformité avec les options qu'il lui est effectivement possible de choisir.

Classer la présence de besoins non satisfaits comme révélatrice d'une lacune d'opportunités peut donc être débattu. Mais plus généralement, c'est le statut des appréciations individuelles qui est en cause.
}

La prise en compte d'opportunités de formations proposées ou imposées au salarié est certes utile à la compréhension du phénomène étudié ; elle demeure néanmoins incomplète et mérite d'être précisée au regard du mode de formation en jeu. En effet, les modes de formation ne sont pas homogènes ni du point de vue du contenu de la formation, ni du point de vue de l'objectif visé, ni encore du point de vue de la transférabilité des savoirs acquis (Hanchane et Lambert, 2003).

Aussi, pour caractériser les libertés réelles d'accès à la formation continue, notre étude se fonde sur cinq fonctionnements : (1) le cours, (2) la FEST (formation en situation de travail), (3) le séminaire, (4) l'autoformation, (5) l'absence de formation ( $c f$. Encadré 2). Ces cinq fonctionnements peuvent être affinés à trois niveaux par des informations portant sur les contraintes et opportunités; en l'occurrence, il s'agit de la perception des salariés sur les conditions qui président à l'entrée ou non dans les diverses voies de formation. L'affinement des fonctionnements résulte donc de la réponse des salariés aux questions suivantes :

\section{Encadré 2 Les quatre modes de formation des salariés}

1. Le stage, ou cours de formation, est le mode de formation continue le plus couramment utilisé par les salariés (64\% des formés). II se déroule avec un formateur ou un intervenant spécialisé, généralement à l'extérieur de l'entreprise.

2. Les formations en situation de travail (FEST) s'organisent avec l'appui d'un tuteur sur le lieu de travail (23\% des formés). Mobilisant les outils de travail du salarié, ces formations peuvent s'apparenter à une simulation de la situation de travail et comporter des moments de production effectifs $\left({ }^{*}\right)$.

3. Les séminaires ( $8 \%$ des formés) font référence à un ensemble de pratiques de formation assez hétérogène (conférences, colloques, salons, foires, etc.). Relativement aux cours et stages, ces situations sont moins fondées sur la didactique que sur l'échange et la discussion.

4. L'autoformation (5 \% des formés) désigne les pratiques de formation où la personne se forme principalement seule (sur son lieu de travail ou à son domicile) en faisant appel à des outils pédagogiques particuliers (support papier, CD-Rom, cassettes vidéo, etc.).

(*) Cette définition exclut les apprentissages informels sur le tas et les mises en situation non organisées d'un point de vue pédagogique (apprentissage par mimétisme « en observant un collègue »).

Source : Enquête Formation Continue 2000. 
- Au cours des 14 derniers mois (ou depuis l'arrivée dans l'entreprise), vous a-t-on proposé une formation que vous avez refusée? (Oui-Non)

- Au cours des 14 derniers mois, avez-vous eu un besoin ou une envie de formation non satisfaits dans un but personnel ou professionnel? (Oui-Non)

- Au cours des 14 derniers mois, votre employeur vous a-t-il imposé les formations que vous avez suivies? (Oui-Non) (cette question ne concerne que les salariés formés).

À partir de ces quatre indicateurs, 36 combinaisons sont possibles, représentant autant de fonctionnements affinés.

Nous avons réalisé une classification ascendante hiérarchique mixte des salariés ( $c f$. Encadré 3). Il en ressort six groupes de salariés ( $c f$. schéma 1) et une diversité des options vis-à-vis de l'ensemble de choix offerts :

- L'absence d'opportunité de formation (classes 1 et 2);

- Des opportunités de formation dans un contexte peu contraint (classe 3 et 4 ) ;
- Des opportunités limitées et marquées pour certains types de formation dans un contexte plus ou moins contraint (classe 5 et 6 ).

\section{Absence d'opportunité de formation...}

Un premier type de salariés se caractérise par l'absence d'opportunités de formation: aucun d'entre eux n'a jamais refusé de formation au cours des 14 derniers mois et aucun d'eux n'en a non plus suivi ( $c f$. tableau 1). Ce premier type concerne plus $60 \%$ des salariés. Mais là où certains expriment des besoins en formation non satisfaits (classe 2), d'autres n'en revendiquent aucun (classe 1).

... et les salariés n'expriment aucun besoin de formation non satisfait : classe 1

Cette première classe concerne la moitié des salariés de l'enquête. La majorité sont ouvriers ( $41 \%$ contre $31 \%$ en moyenne). Un tiers est non diplômé et un tiers possède le certificat d'aptitude professionnelle (Cap) ou le brevet d'études professionnelles (Bep). Cette classe comprend une proportion un peu plus

\section{Encadré 3 \\ Présentation de la méthode de classification utilisée}

Nous avons effectué une classification ascendante hiérarchique mixte qui utilise conjointement la méthode de classification ascendante hiérarchique (CAH) et la méthode d'agrégation autour de centres mobiles. Cette méthode permet de constituer des groupes d'objets homogènes et différenciés avec la garantie de fournir des partitions stables pour des échantillons de taille importante $1>10000$ observations). Le principal avantage de la CAH par rapport aux autres méthodes de classification réside dans une représentation sous forme d'arbre qui met en évidence une information supplémentaire : I'augmentation de la dispersion dans un groupe produit par une agrégation. Pour mettre en œuvre cette CAH mixte, nous avons réalisé au préalable une analyse factorielle des correspondances multiples sur la base des fonctionnements affinés.

Les variables nominales actives qui déterminent les axes factoriels sont le type de formation suivie (versus non-accès), l'expression de besoins non satisfaits, le fait d'avoir pu refuser une formation et le fait d'avoir suivi au moins une formation imposée au cours de la période.

Des variables illustratives ont été ajoutées, afin de décrire les salariés : des variables sociodémographiques (sexe, diplôme, statut, secteur d'activité de l'entreprise, catégorie socioprofessionnelle, taille de l'entreprise, ancienneté, expérience vis-à-vis du marché du travaill), des variables caractérisant l'information relative à la formation continue /connaissance du plan de formation, du congé individuel de formation, et du bilan de compétences, de l'obligation de l'employeur de former ses salariés pour les adapter à leur emploi, connaissance des pratiques de formation d'autres salariés), et des variables caractérisant l'environnement professionnel (nouveaux équipements, nouvelle organisation du travail, aménagement et réduction du temps de travail ). 

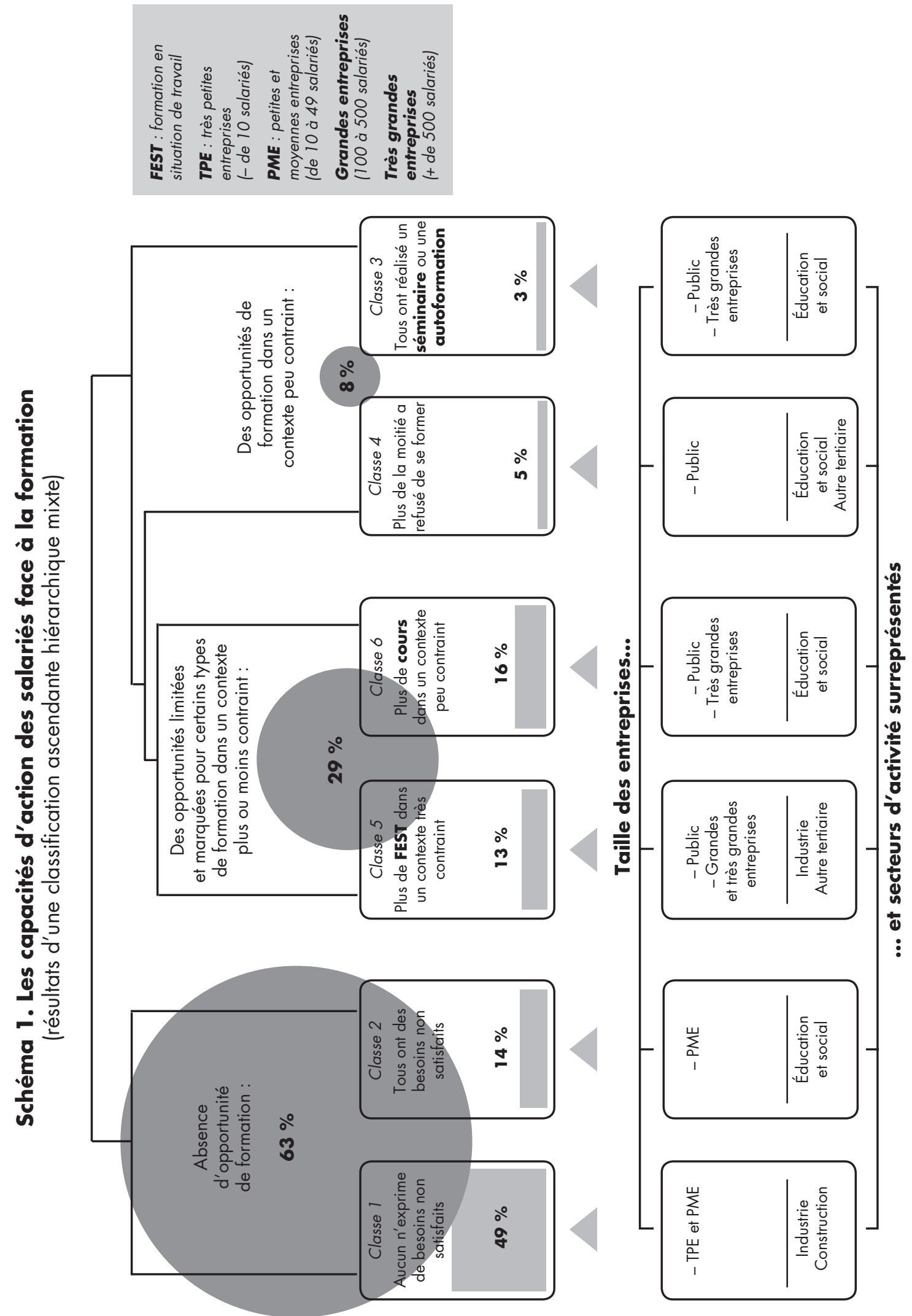
Tableau 1

Description des classes de salariés selon leur capacité d'action face à la formation

\begin{tabular}{|c|c|c|c|c|c|c|c|c|}
\hline & $\begin{array}{c}\text { Classe } 1 \\
49 \%\end{array}$ & $\begin{array}{c}\text { Classe } 2 \\
14 \%\end{array}$ & $\begin{array}{c}\text { Classe } 3 \\
3 \%\end{array}$ & $\begin{array}{c}\text { Classe } 4 \\
5 \%\end{array}$ & $\begin{array}{c}\text { Classe } 5 \\
13 \%\end{array}$ & $\begin{array}{c}\text { Classe } 6 \\
16 \%\end{array}$ & $\begin{array}{c}\text { Ensemble } \\
(\%)\end{array}$ & $\begin{array}{c}\text { Effectif } \\
\text { (en millier) }\end{array}$ \\
\hline \multicolumn{9}{|c|}{ À suivi au moins une formation de type... (au cours des 14 derniers mois) } \\
\hline Cours & 0 & 0 & 0 & 30 & 67 & 76 & 22 & 4276 \\
\hline Séminaire & 0 & 0 & 88 & 0 & 0 & 0 & 3 & 559 \\
\hline $\begin{array}{l}\text { Formation } \\
\text { en situation } \\
\text { de travail }\end{array}$ & 0 & 0 & 0 & 9 & 32 & 20 & 8 & 15078 \\
\hline Autoformation & 0 & ns & 12 & ns & 1 & 4 & 1 & 270 \\
\hline Non formé & $100^{*}$ & 99 & 0 & 60 & 0 & 0 & 66 & 12889 \\
\hline \multicolumn{9}{|c|}{ A refusé une formation qu'on lui avait proposé (au cours des 14 derniers mois) } \\
\hline Oui & 0 & 0 & 8 & 100 & 0 & 0 & 5 & 992 \\
\hline Non & 100 & 100 & 92 & 0 & 100 & 100 & 95 & 18511 \\
\hline \multicolumn{9}{|c|}{ Son employeur lui a imposé au moins une formation (au cours des 14 derniers mois) } \\
\hline Oui & - & ns & 34 & 46 & 100 & 0 & 43 & 2854 \\
\hline Non & - & ns & 66 & 54 & 0 & 100 & 57 & 3760 \\
\hline \multicolumn{9}{|c|}{$\begin{array}{l}\text { A eu un besoin ou une envie de formation non satisfait dans un but personnel ou professionnel } \\
\text { (au cours des } 14 \text { derniers mois) }\end{array}$} \\
\hline Non & 100 & 0 & 68 & 74 & 73 & 72 & 76 & 14790 \\
\hline Oui... & 0 & 100 & 32 & 26 & 27 & 28 & 24 & 4712 \\
\hline \multicolumn{9}{|l|}{$\begin{array}{l}\ldots \text { dans le but } \\
\text { de }\end{array}$} \\
\hline $\begin{array}{l}\text { S'adapter } \\
\text { à son emploi }\end{array}$ & - & 50 & 62 & 52 & 57 & 59 & 53 & 2511 \\
\hline $\begin{array}{l}\text { Obtenir } \\
\text { ou changer } \\
\text { d'emploi }\end{array}$ & - & 24 & ns & 14 & 11 & 12 & 19 & 886 \\
\hline $\begin{array}{l}\text { Obtenir un } \\
\text { diplôme ou une } \\
\text { qualification }\end{array}$ & - & 13 & ns & ns & 12 & 10 & 12 & 559 \\
\hline $\begin{array}{l}\text { Autre } \\
\text { (personnel, sportif, } \\
\text { culturel...) }\end{array}$ & - & 13 & 19 & 21 & 20 & 19 & 16 & 757 \\
\hline \multicolumn{9}{|l|}{$\begin{array}{l}\text {... pour } \\
\text { une formation } \\
\text { de type }\end{array}$} \\
\hline Stage & - & 65 & 75 & 74 & 72 & 77 & 69 & 3252 \\
\hline
\end{tabular}


Tableau 1 (suite)

\begin{tabular}{|c|c|c|c|c|c|c|c|c|}
\hline & $\begin{array}{c}\text { Classe } 1 \\
49 \%\end{array}$ & $\begin{array}{c}\text { Classe } 2 \\
14 \%\end{array}$ & $\begin{array}{c}\text { Classe } 3 \\
3 \%\end{array}$ & $\begin{array}{c}\text { Classe } 4 \\
5 \%\end{array}$ & $\begin{array}{c}\text { Classe } 5 \\
13 \%\end{array}$ & $\begin{array}{c}\text { Classe } 6 \\
16 \%\end{array}$ & \begin{tabular}{|c|} 
Ensemble \\
$(\%)$
\end{tabular} & $\begin{array}{c}\text { Effectif } \\
\text { (en millier) }\end{array}$ \\
\hline FEST & - & 24 & ns & 15 & 20 & 16 & 21 & 1000 \\
\hline Autoformation & - & 4 & ns & ns & ns & ns & 4 & 179 \\
\hline \multirow[t]{2}{*}{ Autre } & - & 7 & ns & ns & ns & 4 & 6 & 281 \\
\hline & 9608 & 2739 & 637 & 943 & 2466 & 3109 & & 19502 \\
\hline
\end{tabular}

ns : non significatif.

FEST : formation en situation de travail.

(*) Note de lecture : parmi les salariés composant la classe 1, aucun n’a réalisé de formation. Pour l'ensemble des 6 classes, les non-formés représentent environ 12889000 salariés.

Source : Enquête Formation Continue 2000.

forte de seniors qui travaillent plus souvent dans des petites entreprises (10 à 50 salariés : $37 \%$ contre $29 \%$ en moyenne).

N'ayant aucune opportunité de formation, ces salariés n'expriment pas pour autant de besoins de formation non satisfaits. Il faut dire qu'ils sont moins bien informés sur la formation, notamment sur l'existence de leurs droits ouverts au titre du congé individuel de formation et du bilan de compétences. De même, ils sont plus nombreux à penser que leur employeur n'est pas obligé de les former pour les adapter à l'évolution de leur emploi. À cela s'ajoute un environnement professionnel peu propice au départ en formation : ils n'ont ni vécu la mise en place de nouveaux équipements, ni celle d'une nouvelle organisation du travail, et les autres salariés de leur entreprise ne suivent pas non plus de formation.

... et les salariés expriment des besoins de formation non satisfaits : classe 2

Cette deuxième classe concerne $14 \%$ des salariés de l'enquête. La répartition en termes de niveau de qualification est relativement proche de la moyenne mais les salariés sont ici plus souvent embauchés sous contrats précaires $(18 \%$ ne sont pas en contrat à durée indéterminée contre $12 \%$ en moyenne). Cette population est également plus jeune que la moyenne (35\% ont entre 25 et 35 ans contre $29 \%$ pour l'ensemble) et plus de la moitié travaille depuis moins de 5 ans dans l'entreprise. Aucune taille d'entreprise ni secteur d'activité n'est ici surreprésenté.
Tout comme la première classe, ce groupe n'a pas d'opportunité de formation. Mais à la différence du premier groupe, les salariés sont ici mieux informés, notamment sur leurs droits au CIF (congé individuel de formation) ou au bilan de compétences, ou encore sur l'obligation de leur employeur de les former pour les adapter à leur emploi. Ces salariés tirent profit de l'information dont ils disposent pour exprimer des besoins en matière de formation, même si ceux-ci ne sont jamais suivis d'effets. Ces besoins témoignent bien souvent de leur insatisfaction quant à l'emploi occupé : ainsi, le désir de changer d'emploi est-il plus souvent invoqué. Afin de réaliser ce souhait, ils sont plus nombreux à vouloir réaliser une formation en situation de travail.

\section{Des opportunités de formation dans un contexte peu contraint...}

En contrepoint, un second type de salariés associe de fortes opportunités de formation dans un contexte peu contraint. Mais là où certains expriment des besoins en formation non satisfaits et accèdent à la formation (classe 3), d'autres refusent des formations (classe 4).

\section{... et des besoins en formation non satisfaits : classe 3}

Ce troisième groupe concerne $3 \%$ des salariés. Les deux tiers n'ont pas suivi de formation imposée par leur employeur, ce qui dénote de faibles contraintes. Parallèlement, ils déclarent, plus que la moyenne 
des salariés, avoir refusé des propositions de formation, ce qui révèle de plus fortes opportunités de formation.

Les cadres sont ici nettement surreprésentés (39\% contre $13 \%$ en moyenne), et la très grande majorité est titulaire d'un bac +2 ou plus. Ces salariés, plus âgés que la moyenne, sont le plus souvent en emploi stable et travaillent depuis longtemps dans leur entreprise $(37 \%$ depuis plus de 15 ans contre $30 \%$ en moyenne). Près de la moitié travaillent dans le secteur public.

Ces salariés, qui disposent par ailleurs d'une bonne information sur la formation et d'un environnement favorable ( 9 sur 10 déclarent que les autres salariés de l'entreprise se forment également), ont tous suivi une formation sous la forme soit d'un séminaire, soit d'une autoformation. Face à ces nombreuses opportunités de formation, ils n'en expriment pas moins des besoins en formation ( $32 \%$ contre $24 \%$ en moyenne). Contrairement à leurs pratiques de formation qui visent plus souvent que la moyenne des objectifs non professionnels (17\% contre $10 \%)$, leurs besoins s'orientent plus volontiers vers le désir de s'adapter à leur emploi ( $62 \%$ contre $53 \%$ ).

\section{... mais des refus de formation : classe 4}

Les personnes qui composent ce groupe ( $5 \%$ des salariés) déclarent toutes avoir refusé au moins une proposition de formation, ce qui témoigne de fortes opportunités de formation. Cette classe se compose notamment de professions intermédiaires $(29 \%$ contre $23 \%$ en moyenne) qui travaillent majoritairement dans le secteur public (48\% contre $31 \%$ pour l'ensemble des salariés). La majorité des salariés de cette classe travaillent depuis plus de 15 ans dans leur entreprise, qui appartient souvent aux secteurs de l'éducation/social ou d'autres secteurs du tertiaire.

Ces salariés, qui bénéficient de nombreuses opportunités de formation, ont également une bonne connaissance de leurs droits en matière de formation, auquel s'ajoute un environnent propice au départ en formation. Dans ce contexte, il est frappant de constater qu'ils refusent de se former : seulement 4 salariés sur 10 ont suivi une formation. De même, les salariés de cette classe déclarent le moins de besoins en formation non satisfaits (hormis la classe 1 qui n'en déclare pas du tout) et lorsqu'ils en déclarent, c'est plus souvent pour un objectif non professionnel ( $21 \%$ contre $16 \%$ en moyenne)

\section{Des opportunités de formation limitées...}

Entre ces cas polaires, $29 \%$ des salariés disposent d'opportunités de formation plus limitées (classe 5 et 6). En effet, bien que formés, tous déclarent ne pas avoir refusé de formation au cours des 14 derniers mois. Par ailleurs, les opportunités dont bénéficient ces salariés sont ciblées sur certains types de formations, avec des contraintes plus ou moins resserrées selon le mode de formation. Là où certains ont de fortes contraintes pour réaliser une FEST (13\%), d'autres en subissent moins pour suivre un cours $(16 \%)$.

\section{... pour des formations en situation de travail dans un contexte très contraint : classe 5}

Cette classe ( $13 \%$ des salariés) se distingue par une caractéristique majeure: tous les salariés déclarent que leur employeur leur a imposé au moins une formation. Les contraintes qui pèsent sur ces salariés sont assez nettes, ce qui n'est pas sans lien avec leurs pratiques de formation, plus souvent orientées vers la FEST ${ }^{12}$ (quatre fois plus nombreuse ici qu'en moyenne).

Les salariés composant cette classe exercent plus souvent une profession intermédiaire (31\% contre $23 \%$ en moyenne). Les grandes entreprises du secteur privé sont surreprésentées ( $22 \%$ contre $14 \%$ en moyenne); elles se situent pour la moitié dans le secteur tertiaire (autre que éducation et social) et pour près d'un quart dans l'industrie.

Comparativement aux autres cas, la formation semble, ici plus qu'ailleurs, subordonnée à des enjeux de mutation de l'entreprise : nouveaux équipements, nouvelle organisation du travail et surtout

\footnotetext{
${ }^{12}$ Hanchane et Lambert (2003) ont montré que la formation en situation de travail est un outil que l'employeur utilise à des occasions particulières et pour des objectifs précis d'adaptation au poste de travail.
} 
mise en place d'une réduction du temps de travail ( $37 \%$ contre $27 \%$ en moyenne cf. tableau 2 ). Ces salariés qui évoluent dans un contexte favorable au départ en formation ( $95 \%$ déclarent que les autres salariés de l'entreprise se forment), ont ici une bonne connaissance de leurs droits mais perçoivent trop souvent la formation comme une nécessité. Aussi expriment-ils, plus souvent que la moyenne, des besoins non satisfaits, et même si les formations qu'ils suivent visent en premier lieu l'adaptation à l'emploi (91\% contre $81 \%$ en moyenne) cela ne semble pas combler leurs besoins en ce domaine (57\% voudraient s'adapter à leur emploi contre $53 \%$ en moyenne). Mais ils sont également $20 \%$ à souhaiter réaliser une formation dans un but non professionnel (contre $16 \%$ en moyenne).

\section{... pour des cours dans un contexte peu contraint : classe 6}

Comparativement au cas précédent, les salariés composant cette classe (16\% des salariés) ont pour particularité de n'avoir jamais suivi de formation imposée. Les opportunités, certes plus limitées, dont bénéficient ces salariés, semblent donc davantage rencontrer leur adhésion. Ce résultat est à mettre au regard de leurs pratiques de formation : ici, $76 \%$ des salariés ont suivi un cours contre $22 \%$ en moyenne. Plus de la moitié sont cadres ou exercent une profession intermédiaire. La part des bac et plus est donc largement surreprésentée. Pour l'essentiel, ces salariés exercent dans le secteur public et les secteurs de l'éducation et du social.

Ils possèdent une bonne connaissance de leurs droits en matière de formation, auquel s'ajoute un environnement propice au départ en formation, comme en témoigne la fréquence des changements organisationnels ou des changements d'équipements. Cependant, les formations suivies ne sont pas toujours en lien direct avec l'emploi occupé : $13 \%$ des salariés désiraient changer d'emploi ou obtenir une qualification contre $9 \%$ dans l'ensemble et $13 \%$ visaient un but culturel, sportifs, etc. contre $10 \%$ en moyenne. Ces opportunités de formation n'empêchent pas les salariés d'exprimer des besoins en formation non satisfaits qui s'orientent plus volontiers vers l'adaptation à l'emploi : $59 \%$ contre $53 \%$ en moyenne.

\section{CAPABILITÉS VERSUS ACCÈS : À LA FOIS PEU... ET BEAUCOUP DE DIFFÉRENCES}

Nous venons d'utiliser l'approche par les capacités comme cadre analytique des situations des salariés face à la formation continue. Les capacités d'action des salariés ont été appréciées au regard des fonctionnements affinés combinant les possibilités de choix et les contraintes sur les choix. Une telle approche se focalise sur la liberté réelle des individus comme base informationnelle de jugement. Comme piste d'évaluation des inégalités, l'approche par les capacités va donc à l'encontre d'un cadre privilégiant l'accès, c'est-à-dire basé une analyse des taux d'accès à la formation qui ne permet pas d'ouvrir la question des latitudes d'action. Dans la perspective qui est la nôtre, il est donc utile de s'interroger sur l'apport des résultats d'une approche par les capacités à ceux d'une analyse plus classique fondée sur l'accès.

\section{Des résultats en apparence semblables...}

Le développement des opportunités est une condition nécessaire de toute politique de capabilités. Or, près des deux tiers des salariés n'en disposent pas. Par ailleurs, la typologie réalisée révèle que pour ceux qui en disposent, le niveau de contrainte qui y est associé varie fortement. Ainsi, l'existence de six types de libertés réelles des salariés face à la formation révèle combien la qualification des salariés, mais aussi l'entreprise, caractérisée par sa taille, son activité, et son contexte, sont des facteurs de conversion puissants des ressources en capacités d'action.

\section{Les capacités d'action} suivent le niveau de qualification

$\mathrm{Si}$ les salariés étudiés sont dans des situations contrastées, on s'aperçoit que les capacités d'action suivent le niveau de qualification. Ainsi, l'ouverture du champ des possibles va croissant avec ce niveau. La classification nous a montré que deux groupes (classe 1 et 2) sont particulièrement désavantagés par rapport aux latitudes d'action qui s'offrent à eux. Ils occupent en effet majoritairement des emplois de 
faible qualification après avoir arrêté leurs études au mieux au niveau du Cap-Bep. De même, le restant de ce groupe rassemble, dans des proportions supérieures à la moyenne, de jeunes salariés précaires. Il existe ainsi un très net décalage entre ces salariés et ceux qui disposent de fortes opportunités de formation dans un contexte faiblement contraint (classe 3 et 4 ). Les classes de ce deuxième type rassemblent une population très homogène, majoritairement constituée de titulaires d'un bac +2 , les cadres y sont très largement surreprésentés, le reste de la population étant composé de professions intermédiaires. Dans une position médiane, on trouve des salariés disposant d'opportunités limitées de formation (classe 5 et 6). Ceux-ci occupent plus souvent une profession intermédiaire, mais la présence d'une proportion de cadres supérieure à la moyenne est aussi à noter. Les latitudes d'action des salariés suivent donc les mêmes clivages observés dans l'accès à la formation (Fournier et al. 2002).

Tableau 2

Description des classes de salariés selon le contexte et les attentes des formés

\begin{tabular}{|c|c|c|c|c|c|c|c|}
\hline & Classe 1 & Classe 2 & Classe 3 & Classe 4 & Classe 5 & Classe 6 & Ensemble \\
\hline \multicolumn{8}{|c|}{ Contexte, information sur la formation } \\
\hline \multicolumn{8}{|c|}{$\begin{array}{l}\text { Mise en place de nouveaux logiciels, matériels informatiques, autres équipements ou techniques de production (au } \\
\text { cours des } 14 \text { derniers mois) }\end{array}$} \\
\hline Oui & 35 & 48 & 58 & 56 & 65 & 62 & 47 \\
\hline Non & 65 & 52 & 42 & 44 & 35 & 38 & 53 \\
\hline \multicolumn{8}{|c|}{ Mise en place d'une nouvelle organisation du travail (au cours des 14 derniers mois) } \\
\hline Oui & 26 & 35 & 40 & 45 & $49 *$ & 41 & 34 \\
\hline Non & 74 & 65 & 60 & 55 & 51 & 59 & 66 \\
\hline \multicolumn{8}{|c|}{ Aménagement ou réduction du temps de travail (au cours des 14 derniers mois) } \\
\hline Oui & 25 & 28 & 26 & 26 & 37 & 29 & 27 \\
\hline Non & 75 & 72 & 74 & 74 & 63 & 71 & 73 \\
\hline \multicolumn{8}{|c|}{ Pense qu'il existe un plan de formation ou un document présentant les formations dans son entreprise } \\
\hline Oui & 46 & 51 & 77 & 79 & 72 & 76 & 58 \\
\hline Non & 54 & 49 & 23 & 21 & 28 & 24 & 42 \\
\hline \multicolumn{8}{|c|}{$\begin{array}{l}\text { Pense avoir droit à un congé individuel de formation (CIF) ou à un congé pour bilan de compétence (BC), pendant } \\
\text { lequel il serait payé }\end{array}$} \\
\hline Oui & 36 & 49 & 60 & 59 & 54 & 58 & 46 \\
\hline Non & 64 & 51 & 40 & 41 & 46 & 42 & 54 \\
\hline \multicolumn{8}{|c|}{$\begin{array}{l}\text { Pense qu'un employeur est obligé de former ses salariés pour les adapter à l'évolution de leur emploi et prévenir le } \\
\text { licenciement économique }\end{array}$} \\
\hline Oui & 73 & 85 & 85 & 83 & 89 & 86 & 80 \\
\hline Non & 27 & 15 & 15 & 17 & 11 & 14 & 20 \\
\hline
\end{tabular}


Tableau 2 (suite)

\begin{tabular}{|c|c|c|c|c|c|c|c|}
\hline & Classe 1 & Classe 2 & Classe 3 & Classe 4 & Classe 5 & Classe 6 & Ensemble \\
\hline \multicolumn{8}{|c|}{ Dans son entreprise, d'autres salariés suivent des formations } \\
\hline Oui & 57 & 67 & 92 & 88 & 95 & 91 & 72 \\
\hline Non & 43 & 33 & 8 & 12 & 5 & 9 & 28 \\
\hline \multicolumn{8}{|c|}{ Les attentes des formés } \\
\hline \multicolumn{8}{|c|}{ A suivi une formation dans le but de } \\
\hline $\begin{array}{l}\text { S'adapter } \\
\text { à son emploi }\end{array}$ & - & ns & 76 & 82 & 91 & 74 & 81 \\
\hline $\begin{array}{l}\text { Obtenir ou } \\
\text { changer d'emploi }\end{array}$ & - & ns & ns & ns & 2 & 5 & 4 \\
\hline $\begin{array}{l}\text { Obtenir un diplôme } \\
\text { ou } \\
\text { une qualification }\end{array}$ & - & ns & ns & ns & 3 & 8 & 5 \\
\hline $\begin{array}{l}\text { Autre (personnel, } \\
\text { sportif, culturel) }\end{array}$ & - & ns & 17 & 12 & 4 & 13 & 10 \\
\hline \multicolumn{8}{|c|}{ Espérait augmenter sa rémunération à l'issue de la formation } \\
\hline Oui & - & ns & 8 & 11 & 11 & 13 & 12 \\
\hline Non & - & ns & 92 & 89 & 89 & 87 & 88 \\
\hline
\end{tabular}

* Note de lecture : $49 \%$ des salariés de la classe 5 déclarent qu'il y a eu la mise en place d'une nouvelle organisation du travail dans leur service ou leur équipe au cours des 14 derniers mois.

Source : Enquête Formation Continue 2000.

\section{Les divers modes de formation témoignent du champ des possibles des salariés}

$\mathrm{Si}$ on tient compte des différences entre modes de formation, on constate que les inégalités face aux opportunités réelles de formation ne sont que renforcées par rapport au niveau de qualification. Plusieurs différences peuvent être soulignées à cet égard.

L'analyse des capacités d'action des salariés formés au regard des modes de formation auxquels ils accèdent révèle que les salariés disposant le plus d'opportunités réelles de formation ont recours aux séminaires ou à l'autoformation (classe 3). Il est nécessaire de distinguer les deux logiques qui accompagnent ces modes de formation. Si l'autoformation concerne souvent le développement personnel et relève d'un investissement personnel, le séminaire s'inscrit davantage dans la politique de formation de l'entreprise. Ainsi, les séminaires témoignent d'une grande marge de liberté des salariés occupant des emplois hautement qualifiés face à la formation en entreprise, tandis que l'autoformation révèle leur latitude d'action dans la sphère privée. À l'opposé, le passage par la FEST s'accompagne de fortes contraintes (classe 5). Tout le travail d'analyse a consisté à comprendre et à reconstruire les circonstances dans lesquelles l'accès différencié à la formation prenait sens. Or, le passage par la FEST est révélateur d'une logique qui lie opportunités de formation spécifiques et fortes contraintes à y participer, pour des salariés qui exercent le plus souvent une profession intermédiaire. Il est rarement question pour ces salariés de refuser ce type de formation et, pour tous, la FEST suivie a été imposée. Entre ces cas bipolaires, on trouve les cours et stages (classe 4 et 6 ) qui, s'ils sont assortis de contraintes, répondent 
davantage à l'intérêt convergent du salarié et de l'employeur.

On ne saurait ignorer les différenciations fortes dans les latitudes d'action qui se traduisent par des accès différenciés aux divers modes de formation. Loin d'être mobilisés aléatoirement, ces derniers témoignent du champ des possibles des salariés. L'approche par les capacités invite à interroger l'hétérogenéité de la formation et, en amont, les différences de champ des possibles pour les divers modes de formation.

\section{L'entreprise comme facteur de conversion environnemental}

La notion de capacité d'action individuelle ne doit pas faire oublier que la liberté de choix est fortement contextualisée. À cet égard, les capacités d'action des salariés dépendent bien souvent de ce que les autres font, c'est-à-dire d'une dimension véritablement collective. L'entreprise apparaît, à cet égard, comme un facteur de conversion central des ressources en capabilité pour la formation.

Par l'effet de la taille de l'entreprise d'abord. Les TPE et PME (très petites entreprises et petites et moyennes entreprises) sont en effet surreprésentées parmi les entreprises qui emploient les classes de salariés dont le champ des possibles est le plus faible (classe 1 et classe 2). Les transformations technologiques ou organisationnelles y sont beaucoup moins fréquentes, le travail n'y change guère et la relation qu'entretient l'entreprise avec la formation tend à être distante. Ainsi, les dépenses de formation ouvrent peu de perspectives d'action aux salariés. Ce n'est pas le cas des grandes entreprises qui offrent des opportunités de formation dans un contexte plus ou moins contraint. Ce n'est pas non plus le cas de la fonction publique où la présence d'opportunités se double de plus faibles contraintes.

Par l'effet du secteur ensuite, qui façonne des opportunités et des contraintes de formation assez spécifiques. Les entreprises du secteur de la construction ou de l'agriculture n'offrent quasiment pas d'opportunités de formation. À la différence du précédent, les entreprises du secteur industriel offrent davantage d'opportunités de formation à leurs salariés, mais elles sont arrimées à un type de formation spécifique, la FEST, et les contraintes de participation y sont fortes. En revanche, dans le secteur de l'éducation et $\mathrm{du}$ social, particulièrement représenté dans les classes 3, 4 et 6, les opportunités de formation sont plus importantes et les contraintes moins pesantes.

Ainsi, si l'analyse conduite apparaît fortement centrée sur le salarié, les capacités d'action articulent néanmoins dimension personnelle et collective. Parmi les salariés dont les opportunités réelles de formation sont les plus faibles (classe 1 et 2), près de la moitié déclarent que les autres salariés de leur entreprise ne se forment pas non plus. La mise en place de nouveaux équipements y est plus rare, l'organisation du travail ne change guère, l'information sur la politique de formation y est moins connue. A contrario, parmi les salariés aux champs des possibles les plus larges (classe 5 et 6), la quasi-unanimité déclare que les autres salariés de l'entreprise se forment, les obligations des employeurs en matière de formation sont mieux connues, tout comme les droits des salariés dans ce domaine. Quant à l'activité de travail, des évolutions se donnent plus souvent à voir soit par l'introduction de nouveaux équipements, soit par une nouvelle organisation. Sur ces point, ces salariés rejoignent ceux qui disposent d'opportunités de formation assorties de contraintes (classe 3 et 4 ) dont plus de $80 \%$ déclarent que les actions de formations concernent l'ensemble des salariés de leur entreprise.

Ainsi, les résultats de l'approche par les capacités d'action rejoignent ceux issus de l'analyse en termes d'accès au regard des caractéristiques des entreprises (Gauthier et Marion, 2000).

\section{... mais des interprétations bien distinctes}

Certes, les résultats obtenus à partir de notre tentative d'opérationnalisation de l'approche de Sen s'accordent avec des résultats assez classiques observés grâce à une analyse de l'accès à la formation continue. Pourtant, changer les modes de description conduit à des interprétations différentes de celles qui seraient basées sur la seule utilisation des taux d'accès. Ainsi, comme le souligne Farvaque (2007) «les résultats peuvent s'apparenter, mais la grille de 
lecture n'est pas la même». C'est ce que nous voudrions développer à présent.

\section{Autonomie individuelle contre paternalisme bienveillant}

D'un point de vue normatif, l'approche par les capacités se distingue d'une approche en termes d'accès en mettant l'accent sur le rôle de la liberté réelle. Comme le rappelle Farvaque (2007) : «En insistant sur les capabilités de quelqu'un plutôt que sur ce qu'il réalise, l'approche valorise l'autonomie individuelle (par rapport à toute hétéronomie consistant à imposer des valeurs, voire par opposition à un paternalisme fut-il bienveillant). » Chez Sen, c'est la liberté réelle de chacun qui doit être maximisée. Il ne s'agit donc pas de valoriser un égal accès à la formation, mais de faire en sorte que la liberté réelle soit garantie pour tous. Les valeurs promues sont fondamentalement différentes. En adoptant une approche par les capacités, on valorise la «liberté réelle de tous par la reconnaissance de la pluralité des points de vue légitimes» (Bonvin, 2005). Une approche centrée sur l'accès à la formation ne permet pas d'aborder cette problématique.

D'un point de vue descriptif, l'approche par les capacités suppose de mesurer les libertés réelles et non les résultats en termes d'accès. Il faut le souligner, l'accès différencié à certains types de formation ou le non-accès à la formation peuvent résulter de la présence ou de l'absence de liberté de choix. Raisonner en termes d'accès ne permet pas de saisir cette distinction fondamentale. Le diagnostic requiert donc d'être porté en amont sur le terrain des capacités d'action des personnes face à la formation et non sur le terrain de l'accès.

Mais modifier la base informationnelle de jugement conduit aussi à des prescriptions différentes de celles qui se fonderaient sur l'analyse des taux d'accès. L'approche par les capacités considère que l'action publique doit restaurer ou augmenter les capacités d'action des personnes face à la formation en agissant sur le niveau des ressources et les facteurs de conversion. Une distribution de droits égaux pour la formation serait certainement le point de départ, mais devrait être accompagnée de procédures permettant leur conversion effective en libertés réelles d'action.
En tout état de cause, il ne s'agirait pas de garantir un égal accès à la formation pour tous.

Ce n'est donc pas la même chose d'évaluer la capabilité de formation et l'accès à la formation. Ce qu'il faut donc souligner ce n'est pas le fait que les deux approches sont semblables, mais bien que l'approche par les capacités apporte une information complémentaire quant aux marges de manœuvres. Que peut-on en conclure alors sur l'observation de caractéristiques communes entre les salariés qui n'accèdent pas à la formation et ceux qui ont les plus faibles capabilités ?

\section{Manque d'appétence pour la formation ou manque d'opportunités?}

L'appétence pour la formation n'est pas clairement définie dans l'Accord national interprofessionnel du 20 septembre 2003. Pourtant, les objectifs énoncés dans son préambule visent explicitement à " réduire les inégalités d'accès et favoriser l'appétence à la formation du plus grand nombre». Cette notion ambiguë ne repose pas davantage sur un concept juridique ou scientifique déterminé, d'où son ambiguité. Elle est néanmoins porteuse d'une responsabilisation du salarié dans la non-participation à la formation. Loin de se résumer à un problème de terminologie, la question de l'appétence pour la formation soulève de véritables enjeux en termes de partage de responsabilités entre institutions publiques, entreprises et individus.

Il faut rappeler que, chaque année, 5 millions de salariés sont formés dans le cadre du plan de formation, contre 35000 qui bénéficient en moyenne d'un CIF (Möbus, 2006). Autrement dit, pour plus de $99 \%$ des salariés, le cadre légal ne prévoit pas une initiative du salarié. Par ses obligations de dépenses et son obligation d'adapter les salariés à leur poste de travail, l'entreprise propose une offre de formation dans le cadre du plan de formation. Le salarié réagira donc conditionnellement à l'offre qui lui sera proposée et aux contraintes imposées. Les refus des salariés visà-vis des offres de formation de leur entreprise pourraient être considérés comme un manque d'appétence pour la formation. Les autres cas de figure ne peuvent pas être imputés aux salariés.

Ainsi, si l'appétence se rattache au choix effectué par le salarié conditionnellement à l'ensemble de ses 
opportunités réelles, il importe de confronter ce choix aux résultats en termes d'accès. Or, les résultats obtenus révèlent, à quelques exceptions près, que la non-participation à la formation relève davantage d'un manque d'opportunités que d'un manque d'appétence. En effet, parmi les $62 \%$ de salariés qui n'accèdent pas à la formation, $59 \%$ n'ont strictement aucune opportunité, et seulement $3 \%$ opposent un refus aux offres de formation émanant de leur entreprise. Que reste-t-il alors de l'autonomie et du consentement libre et éclairé des salariés face à la formation? La confrontation des opportunités réelles de formation et de l'accès effectif est déjà révélatrice de la faiblesse des capacités d'action des salariés en marge de la formation. C'est en effet moins leur inappétence à la formation que la faiblesse de leur latitude d'action qui est en cause.

Ce constat est un résultat majeur pour le présent travail, qui va à l'encontre de craintes fréquemment exprimées selon lesquelles les salariés les plus éloignés de la formation continue auraient moins d'appétence pour celle-ci.

Reste à savoir comment se compose ce sous-groupe de la classe 4, cette infime minorité de salariés qui ne se saisissent pas des opportunités de formation qui leur sont offertes. Il rassemble une population féminine, plus souvent ouvrière, qui a arrêté ses études à la fin de la scolarité obligatoire, au niveau du certificat d'études professionnelles. Les plus de 50 ans sont ici surreprésentées. Cette logique de retrait face à la formation traduit un véritable blocage de leur trajectoire professionnelle : celles qui ont déjà eu à se former dans les périodes précédant l'observation ont deux fois moins souvent vu leur rémunération augmenter à l'issue de la formation. Au-delà des opportunités de formation se pose donc la question des opportunités de développement professionnel.

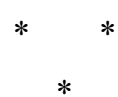

Il s'agissait d'appréhender, au travers de cette étude, les capacités d'action des salariés face à la formation. Mais au-delà de cette question, comme le notait Salais (2006) à propos de l'analyse de la politique sociale et de l'emploi en Europe, «le thème central de l'approche par les capacités est la construction d'une sécurité active pour faire face aux transformations du travail et à l'incertitude économique » (Salais, 2006, p. 16). Ainsi, l'approche par les capacités ne se résume pas à l'exercice effectif d'une liberté de choix face à la formation. Celle-ci n'est pas seulement une fin mais aussi un moyen permettant d'accroître les libertés des personnes dans le développement de leur parcours professionnel.

Pour près de la moitié des salariés, les besoins de formation non satisfaits relèvent d'une autre logique que l'adaptation au poste de travail, alors que les opportunités classiques face à la formation visent cet objectif d'adaptation. Ce scénario semble prévaloir dans la plupart des situations observées. La quête d'une dynamique de formation se traduisant par le développement des compétences est généralement vaine. Il n'y a guère que parmi les salariés ayant de fortes opportunités de formation dans un contexte peu contraint que ce type de besoins se ressent le moins, les cadres et professions intermédiaires sont ici surreprésentés. Mais globalement, le bilan est sans équivoque: les latitudes d'action offertes aux salariés ne leur permettent pas d'être acteurs du développement de leur trajectoire ; ceci est particulièrement vrai pour les emplois faiblement qualifiés. Analyser les opportunités de formation en distinguant les différents objectifs de formation revient à souligner le point critique, critique parce qu'il pose le problème de l'accès à la formation valorisante pour le salarié dans le développement de son parcours professionnel.

Si la logique de capabilité est associée à une exigence de responsabilisation du salarié quant à sa carrière et à sa formation, «il importe que ce dernier dispose en retour des outils permettant d'assumer une telle responsabilité » (Zimmermann, 2005). Or, à la veille de la mise en application de la loi du 4 mai 2004, force est de constater que c'est ici que le bât blesse. Peu d'entreprises offrent des latitudes d'action dans le développement des compétences au regard de l'ampleur des opportunités de formation visant l'adaptation à l'emploi. Cela tient avant tout au contexte institutionnel, c'est-à-dire au déficit de droits des salariés à se former dans le cadre de leur entreprise. Dans le vocabulaire de Sen, on parlera d'un déficit de ressources. 
Certes, les nouvelles dispositions introduites par la loi du 4 mai 2004 procèdent d'une augmentation des ressources, mais on sait peu de choses sur les changements qu'elles produiront en termes de capacité d'action des salariés. Alors que la loi entend créer un espace de négociation entre employeur et salarié, pour les formations visant le développement des compétences, «le risque existe, ainsi d'aboutir, en pratique, à un détournement $d u$ DIF [...] vers des formations d'adaptation relevant du plan de formation » (MaggiGermain, 2004). L'étude «Regards croisés sur la formation», analysant le cas d'entreprises ayant anticipé sur la nouvelle loi nous en procure un bon exemple: "La classification des formations en trois groupes (adaptation, métier et compétences), le dernier groupe faisant l'objet d'un co-investissement, donne lieu à des interprétations qui ne sont pas toujours favorables aux salariés. » (Perez et Vero, 2006) Il reviendrait alors aussi aux acteurs collectifs de produire des garanties collectives, notamment en matière de gestion des carrières, comme facteurs de conversion des ressources en capacités d'action, via des plans de développement professionnels ou des revues de parcours professionnels, par exemple.

\section{Bibliographie}

Berton F. et Podevin G. (1991), « Vingt ans de formation professionnelle continue: de la promotion sociale à la gestion de l'emploi », Formation Emploi, $\mathrm{n}^{\circ} 34$, pp. 14-34.

Bonvin J.-M. (2005), « Sen et la démocratie », l'Économie Politique, $\mathrm{n}^{\circ} 27$, pp. 24-37.

Charlon-Dubar E., Dubar C., Engrand S., Feutrie M., Gadrey N., Vermelle M.-C. (1990), «Le salarié confronté à l'offre de formation. Trajectoire personnel, identité professionnelle et logique d'entreprise»,

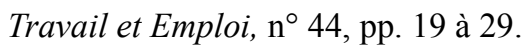

Demazière D. et Dubar C. (1997), Analyser les entretiens biographiques. L'examen de récits d'insertion, Nathan, $350 \mathrm{p}$.

Dupray A. et Hanchane S. (2001), « Les effets de la formation continue en entreprise sur la mobilité et le salaire des jeunes », Formation Emploi, n 73.

Farvaque N. (2005), Action Publique et approche par les capacités : une analyse des dispositifs et trajectoires d'insertion, thèse, Polytechnicium Marnela-Vallée, sous la direction de R. Salais.
Farvaque N. (2007), «"Faire surgir des faits utilisables". Le caractère opérationnel de l'enquête sur les capacités en question ", in de Munck J. et Zimmermann B. (Éds), L'enquête sur les capacités, Raisons pratiques 18, Éditions de l'EHESS, novembre 2007 (à paraître).

Fournier C., Lambert M. et Perez C. (2002), «Les français et la formation continue. Statistiques sur la diversité des pratiques », Document Céreq, n 169 , Série Observatoire, novembre.

Fournier C. (2001), «Hommes et femmes salariés face à la formation continue. Des inégalités qui reflètent les niveaux de qualification et les conditions familiales », Céreq-Bref, n 179, 4 p.

Fournier C. (2003), «La formation continue des salariés du privé à l'épreuve de l'âge », Céreq-Bref, $\mathrm{n}^{\circ} 193,4 \mathrm{p}$.

Fournier C. (2004), « Aux origines de l'inégale appétence des salariés... », Céreq-Bref, n 209, 4 p.

Gauthier C. et Marion I. (2000), « La formation professionnelle continue financée par les entreprises » Documents Céreq, n 172, 89 p. 
Gelot D. (2006), « Le rôle de l'encadrement intermédiaire dans la formation en entreprise », Travail et Emploi, $\mathrm{n}^{\circ} 107$, juillet-septembre, pp. 47-58.

Goux D. et Maurin E. (2000), «Returns to firmprovided training: evidence from French workerfirm matched data », Labour economics, $\mathrm{n}^{\circ} 7$, pp. 1-19.

Goux D. et Zamora P. (2001), «La formation en entreprise continue de se développer ", Insee Première, $\mathrm{n}^{\circ} 759$.

Géhin J.-P. et Verdier E. (1988), «Après 16 ans d'obligation légale, la formation professionnelle contnue dans les entreprises ", Actualités de la formation permanente, $\mathrm{n}^{\circ} 93$.

Lambert M. et Hanchane S. (2003), «La variété des modes de formation : usages et enjeux », Formation Emploi, $\mathrm{n}^{\circ}$ 81, pp. 51-66.

Lambert M. et Perez C. (2004), «Stratégies de recours et d'usage de la formation des "nouveaux recrutés": une comparaison public-privé ", in Les déterminants des entrées dans les fonctions publiques: parcours du combattant ou pis-aller?, Paris, ministère de la Recherche, pp. 109-141.

Le Clainche C. (1994), « Niveau de vie et revenu minimum : une opérationalisation du concept de Sen sur données françaises », Cahier de Recherche $d u$ CRÉDOC, $\mathrm{n}^{\circ}$ 57, avril.

Lainé F. (2003), « Les seniors et la formation continue : un accès en général limité mais avec de grandes différences selon les situations professionnelles », Premières informations, premières synthèses, ${ }^{\circ} 12.1$, Dares, mars.

Maggi-Germain N. (2004), « La formation professionnelle continue entre individualisation et personnalisation des droits des salariés », Droit Social, mai, pp. 482-493.

Möbus M. (2006), «Le développement des compétences dans le travail temporaire en France. Approches et dispositifs", Notes Emploi Formation, Céreq, à paraître.
Perez C. et Thomas G. (2004), « La formation continue dans les trajectoires d'emploi précaires », Notes Emploi Formation, $\mathrm{n}^{\circ} 19$.

Perez C. et Vero J. (2006), «L'accès à la formation en entreprise face à l'évolution des modes de gestion de la main-d'œuvre», Travail et Emploi, $\mathrm{n}^{\circ}$ 107, juillet-septembre, pp. 59-71.

Personnaz E. et Vernoux-Marion I. (2006), « La formation continue : un temps pour mobiliser les salariés autour de valeurs et de pratiques communes », Bref-Céreq, avril, n² 229, 4 p.

PNUD (1998), Rapport Mondial sur le développement humain 1998, De Boeck University.

Salais R. et Villeneuve R. (Éds) (2006), Développer les capacités des hommes et des territoires en Europe, Éditions de l'Anact, Lyon, 456 p.

Sen A. K. (1980), "Equality of What ?" in Mc Murrin (éd), Tanner Lectures on Human Values, Cambridge University Press. Réimpression in Sen A.K (1982a). Traduction Française « Quelle Égalité ? », Éthique et économie. Et autres essais, Presses Universitaires de France, 1999.

Sen A. K. (1985), Commodities and Capabilities, North Holland, 89 p.

Sen A. K. (1992), Repenser l'inégalité, Seuil, 2000, $282 \mathrm{p}$.

Sen A. K. (1999), Éthique et économie, PUF, 364 p.

Vero J. (2002), Mesurer la pauvreté à partir des concepts de biens premiers, de réalisations primaires et de capabilités de base. Le rôle de l'espace d'information dans l'identification de la pauvreté des jeunes en phase d'insertion professionnelle, thèse, EHESS, sous la direction de P. Werquin, $281 \mathrm{p}$.

Zimmerman B. (2000), «Logiques de compétences et dialogue social », Travail et Emploi, ${ }^{\circ}$ 84, p. 5

Zimmermann B. (2006), « Employabilité et logique de compétences » in Salais R., Villeneuve R. (Éds), Développer les capacités des hommes et des territoires en Europe, Éditions de l'Anact, pp. 74-93. 
Résumé

\section{Formation continue : quelles capacités d'action des salariés? L'approche par les capacités comme grille de lecture}

Marion Lambert et Josiane Vero

Cet article analyse les inégalités entre salariés en matière de formation continue selon l'approche par les capacités développée par Sen. II éclaire la question des inégalités face à la formation continue dans une double direction. Premièrement, en se centrant sur la liberté réelle de choix des salariés, il invite à dépasser l'opposition trop schématique entre formés et non-formés. Loin d'être mobilisés aléatoirement, les divers modes de formation reflètent le champ des possibles des salariés. Dès lors, considérer la formation comme une catégorie homogène ne rend pas compte de la réalité. Deuxièmement, il éclaire les latitudes d'action des salariés face à la formation. Les caractéristiques des salariés ayant le champ des possibles le plus restreint sont semblables à celles, bien connues, des salariés qui n'accèdent pas à la formation. Ainsi, l'argument du manque d'appétence pour la formation n'apparaît pas pertinent.

\section{Mots-clés}

Approche par les capabilités, formation professionnelle en entreprise, accès à la formation professionnelle Journal of Economic Literature: D 63, I 29, M 12 


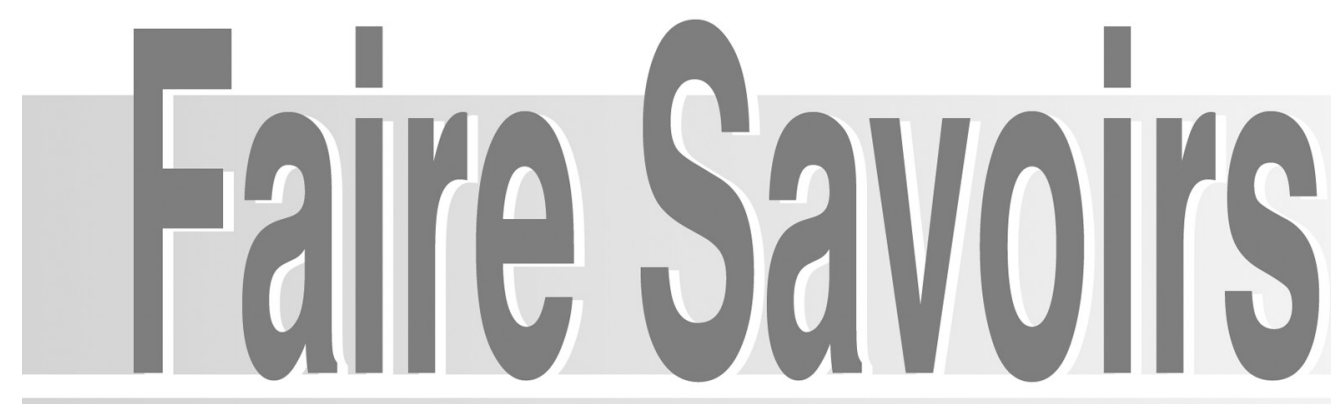

Sciences humaines et sociales en région PACA

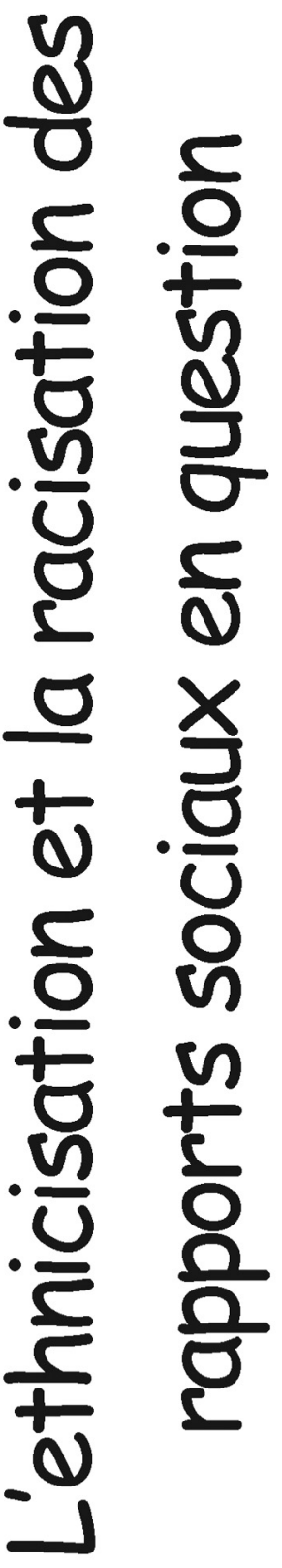

Somemeaire

\section{L'ethnicisation et la racisation des rapports sociaux en question}

3 Présentation du dossier

\section{Dossier 3}

Le paradigme de l'ethnicité. 15 Développements en France et perspectives

La politique du logement social est-elle raciste? L'exemple marseillais - Valérie Sala Pala

Racialisation? Le cas de la colorisation coloniale des rapports sociaux - Jean-Luc Bonniol

Les économistes et l'analyse du travail servile (1750-1850) - André Legris

61

L'ethnicisation/racialisation des rapports interpersonnels - Michel Piolat

\section{Etude Régiomale}

73 L'hébergement des demandeurs d'asile dans les Alpes-Maritimes - Gilles Frigoli

\section{Thèses}

85 Le rôle des mémoires collectives dans la construction du sentiment d'appartenance territoriale. Le cas du Parc naturel régional du Verdon

$$
\text { - Mathieu Leborgne }
$$

91 Migrantes et migrants dans les emplois domestiques en France et en Italie: construction sociale de la relation de service au croisement des rapports sociaux de sexe, de race et de classe - Francesca Scrinzi

\section{Lecture}

99 Bernard Conein, (2005). Les sens sociaux. Trois essais de sociologie cognitive. Paris: Economica - Jacques Guilhaumou

Association Méditerranéenne d'Animation, de Rencontres et d' Echanges en Sciences de l' homme et de la société wWw.amares.org 